\title{
Alveolar rhabdomyosarcoma with massive dis- seminated intravascular coagulopathy treated with systemic chemotherapy
}

\author{
Byung Gyu Yoon, MD', Hee Jo Baek, MD, PhD', Burm Seok Oh, MD', Dong Kyun Han, MD, PhD', Yoo Duk Choi, MD², Hoon Kook, MD, PhD', \\ Departments of ${ }^{1}$ Pediatrics and ${ }^{2}$ Pathology, Chonnam National University Hwasun Hospital, Chonnam National University Medical School, Hwasun, Korea
}

It is uncommon for pediatric patients with rhabdomyosarcoma to present with clinical and/or laboratory features of disseminated intravascular coagulation (DIC). We report a case of metastatic alveolar rhabdomyosarcoma with severe bleeding because of DIC in a 13-year-old boy. He experienced persistent oozing at the site of a previous operation, gross hematuria, and massive epistaxis. Two weeks after initiating combination chemotherapy consisting of vincristine, doxorubicin, and cyclophosphamide, the patients' laboratory indications of DIC began to resolve. During this period, the patient received massive blood transfusion of a total of 311 units (26 units of red blood cells, 26 units of fresh frozen plasma, 74 units of platelet concentrates, 17 units of single donor platelets, and 168 units of cryoprecipitate), antithrombin-III and a synthetic protease inhibitor. Despite chemotherapy and radiation therapy, he died 1 year later because of disease progression. In children with metastatic rhabdomyosarcoma and massive DIC, prompt chemotherapy and aggressive supportive care is important to decrease malignancy-triggered procoagulant activities.

Key words: Rhabdomyosarcoma, Disseminated intravascular coagulation, Chemotherapy

\section{Introduction}

Disseminated intravascular coagulation (DIC) is a systemic thrombohemorrhagic disorder with variable pathophysiology. The underlying diseases causing DIC include obstetric accidents, intravascular hemolysis, septicemia, viremia, burns, trauma, acute liver disease, and malignant disease ${ }^{1)}$. There is a causal relationship between DIC and the presence of malignancy ${ }^{2)}$. The incidence of DIC was reported on 7\% of the patients with solid tumor and 15\%$20 \%$ of the patients with acute leukemia ${ }^{3,4}$. DIC in pediatric solid tumor has been reported with disseminated neuroblastoma, nephroblastoma, infantile fibrosarcoma, and multi-system Langerhans cell histiocytosis ${ }^{5}$. The pediatric patients with rhabdomyosarcoma presenting with DIC at diagnosis are rare and are mainly seen with the widespread alveolar rhabdomyosarcoma metastatic to bone marrow $(\mathrm{BM})^{3)}$. Clinically, DIC in cancer is less fulminant than the types of DIC seen in sepsis and trauma. Also, chronic and systemic activation of coagulation can progress subclinically, and this progression may lead to exhaustion of coagulation factors and platelets. Therefore, bleeding may be the first clinical symptom of DIC in a patient with cancer²).

Here, we describe an unusual case with acute bleeding and DIC, which were severe and very difficult to control, with the presenting manifestation of alveolar rhabdomyosarcoma metastatic to BM. Early administration of chemotherapy and vigorous supportive care could control the massive bleeding and DIC.
Corresponding author: Hee Jo Baek, MD, PhD Department of Pediatrics, Chonnam National University Hwasun Hospital, Chonnam National University Medical School, 322 Seoyang-ro, Hwasun 58128, Korea

Tel: +82-61-379-7695

Fax: +82-61-379-7697

E-mail: swan93@|ycos.co.kr

Received: 25 October, 2013

Revised: 13 May, 2014

Accepted: 16 May, 2014
Copyright (C) 2015 by The Korean Pediatric Society

This is an open-access article distributed under the terms of the Creative Commons Attribution NonCommercial License (http://creativecommons.org/ licenses/by-nc/3.0/) which permits unrestricted noncommercial use, distribution, and reproduction in any medium, provided the original work is properly cited. 


\section{Case report}

A 13-year-old boy was admitted in January 2011, due to an uncontrolled bleeding at the operation site of the right thenar muscle. The swelling of the right thenar muscle developed after a fall on August 2010 (Fig. 1A, B). However, no treatment was given under the impression that the swelling was a hematoma. After showing no improvement for over 5 months, excision of this lesion was attempted at a private orthopedic surgery clinic. Before marginal excision, the patient had no history of bleeding tendency and normal coagulation screening tests. He underwent two additional operations at different hospitals for the control of bleeding, but the bleeding at the operation site continued.

When a pathologic finding of the mass suggested a diagnosis of malignancy, he was transferred to our hospital for further management. At the admission, the patient's general state was poor with signs of pallor, weakness, bruising at the intravenous site, and purpura on the operation site over the right thenar muscle with continuous bleeding (Fig. 1C). In the physical examination, there was a $2 \times 2 \mathrm{~cm}$-sized lymph node (LN) in the right axillary area and $1 \mathrm{~cm}$-sized hepatosplenomegaly. Laboratory tests revealed markedly decreased platelet and hemoglobin levels and features suggestive of DIC (white blood cell, 9,900 / $\mu \mathrm{L}$; hemoglobin, $4.8 \mathrm{~g} / \mathrm{dL}$; platelets, 28,000 / $\mu \mathrm{L}$; prothrombin time, 1.47 international normalized ratio; activated partial thromboplastin time, $45.4 \mathrm{sec}$; fibrinogen, $87 \mathrm{mg} / \mathrm{dL}$; fibrin/fibrinogen degeneration product, $656.4 \mu \mathrm{g} / \mathrm{mL}$; and D-dimer, $16.17 \mathrm{mg} / \mathrm{L})$. The coagulation factors II, VII, IX, fibrinogen, and von willebrand factor were all normal. The factors V and VIII were 30\% (normal, 60\%-140\%) and 40\% (normal, 50\%-150\%), respectively. 18-Fluoro-2-deoxyD-glucose positron emission tomography scan showed hypermetabolic lesions (maximum standardized uptake value [SUVmax], 2.7) along the rim of a previous mass in the right thenar muscle and conglomerated hypermetabolic lesions (SUVmax, 5.3) in the right axillary space. It suggested post-operative inflammation in the right thenar muscle and metastatic lymphadenopathy in the
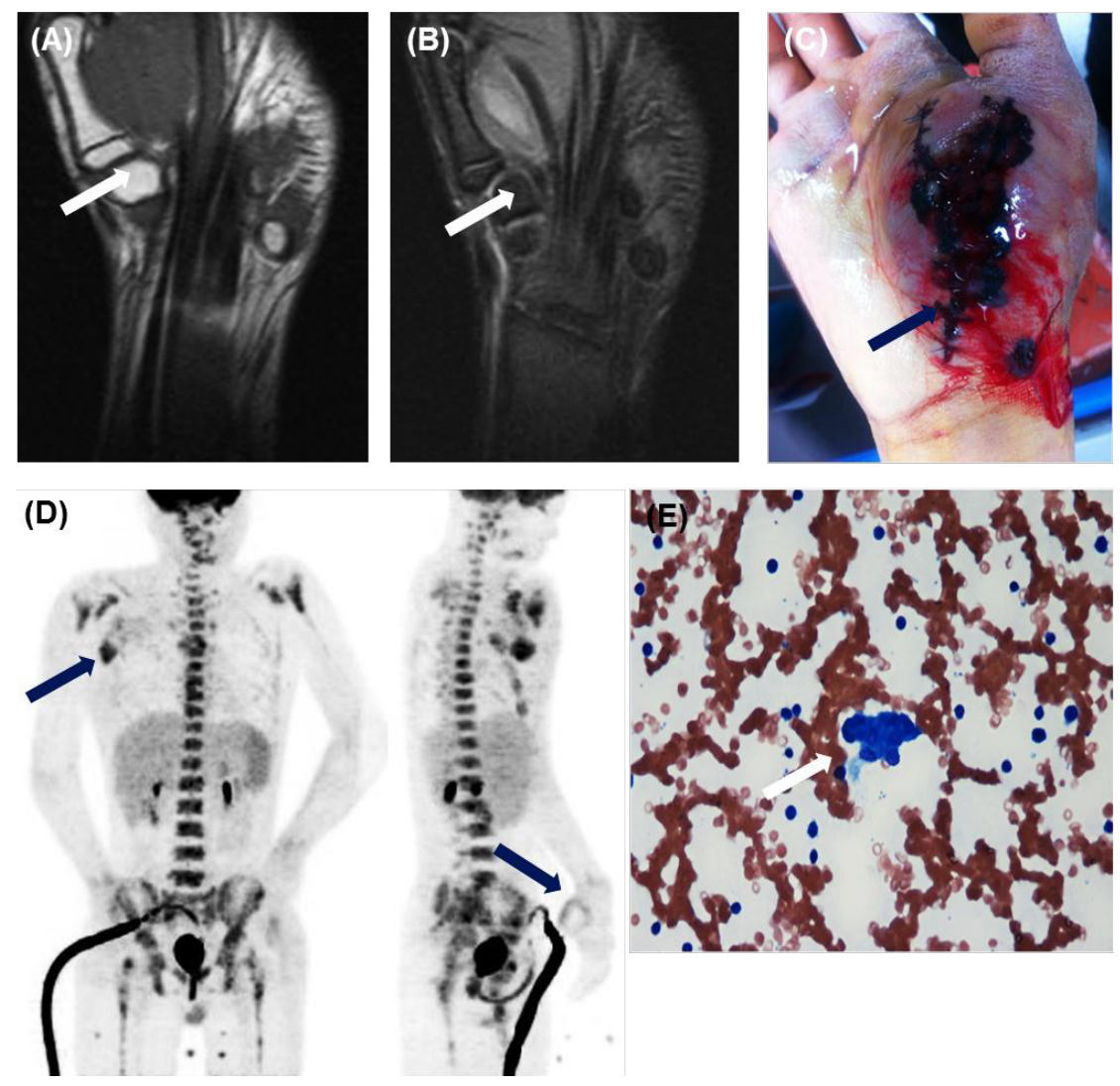

Fig. 1. Findings at initial presentation. (A, B) Magnetic resonance imaging revealed a $5 \times 3.6 \times 5-\mathrm{cm}$ well-defined mass in the plantar aspect of the right thenar muscle (arrows). (A) A T1-weighted image with iso-signal intensity. (B) A T2-weighted image with heterogeneous high signal intensity. (C) At admission, bleeding at the site of the previous operation continued. (D) ${ }^{18}$ Fluoro-2deoxyglucose-positron emission tomography revealed metastatic lymphadenopathy (maximum standardized uptake value [SUVmax], 5.3) in the right axillary space as well as postoperative inflammatory changes (SUVmax, 2.7) in the right thenar muscle (arrows). (E) Bone marrow smear showed small round blue cells, suggesting a nonhematopoietic malignancy $(\mathrm{H} \& \mathrm{E}, \times 400)$. 
right axillary space (Fig. 1D).

On the 3rd hospital day (HD), despite of the repeated blood product transfusions, the massive bleeding from the operation site and the intravenous access site worsened, and a gross hematuria developed. On the 4th $\mathrm{HD}$, the pathologic findings of the mass were reported as alveolar rhabdomyosarcoma with positive immnunohistochemical stains of myognesin, desmin, and MyoD and with negative resection margin. The patient was transferred to the intensive care unit, and chemotherapy consisting of vincristine, doxorubicin, and cyclophosphamide (VAC) was administered. Also, repeated transfusions, synthetic protease inhibitor, and substitution of antithrombin-III were administered.

On the 10th HD, a massive epistaxis led to hematemesis of approximately $1.5 \mathrm{~L}$ of blood, and it required transfusions of 4 units of red blood cells (RBCs), 9 units of platelet concentrates (PCs), 1 unit of fresh frozen plasma (FFP), and 8 units of cryoprecipitate, with two times of cauterization. Two weeks after the chemotherapy was started, the laboratory findings of DIC showed improvement (Fig. 2), so a BM section biopsy and an axillary LN biopsy were performed. The histologic finding of BM (Fig. 1E) and axillary LN revealed involvement of rhabdomyosarcoma.

During the initial 20 days of admission, the patient received total blood transfusions of 311 units (26 units of RBC, 26 units of FFP, 74 units of PC, 17 units of single donor platelets, and 168 units of cryoprecipitate) for the control of the DIC and bleeding. On the 28th HD or three weeks after the start of chemotherapy, the patient was discharged.
The patient received a combination chemotherapy consisting of VAC every 3 weeks. At 9th week after the start of chemotherapy, the size of right axillary LN decreased from $3.6 \mathrm{~cm}$ to 2.1 $\mathrm{cm}$. At 21st week after the start of chemotherapy, the BM showed disappearance of rhabodyosarcoma and negative immunostaining for desmin or myogenin. For the planned high-dose chemotherapy with autologous peripheral blood stem cell (APBSC) transplantation, two rounds of APBSC collections were performed. Unfortunately, however, at the 27th week of chemotherapy, the patient was found to have a nodule in the right forearm that was increasing in size for over 2 weeks, and the biopsy revealed it as an alveolar rhabdomyosarcoma. Despite of the salvage chemotherapy consisting of ifosfamide, carboplatin, etoposide, and palliative radiation therapy (right axilla, arm, thenar muscle, and liver, 36 Gy), the patient died 5 months later from the disease progression to the liver and the brain.

\section{Discussion}

The solid malignancies are rare causes of DIC, and the mechanism is unclear. Recent studies show that the solid tumors express different procoagulant molecules including tissue factor (TF), which binds to factor VII(a) to activate the factors IX and X, and to the cancer procoagulant, which is a cysteine protease with factor Xactivating properties. Alternatively, most tumors induce a hypofibrinolytic state, due to high levels of fibrinolytic inhibitor such as

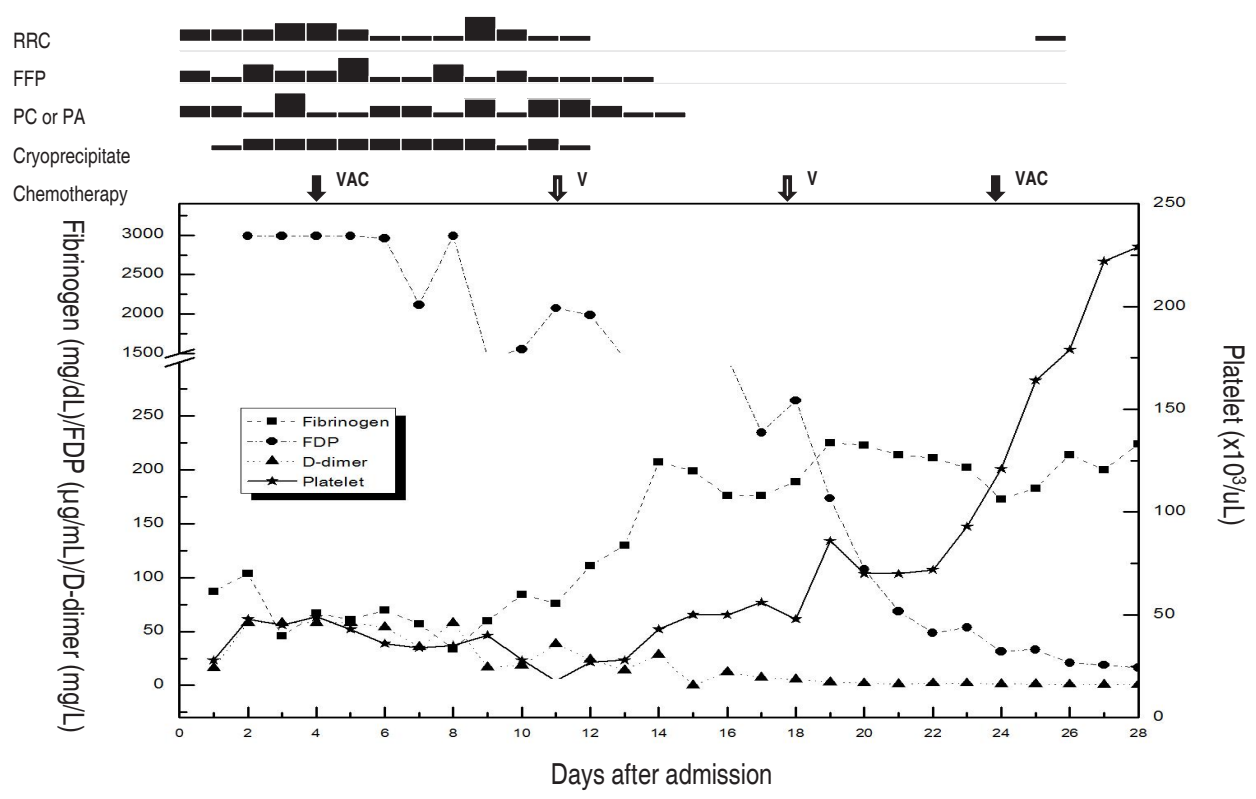

Fig. 2. Clinical course of the patient. After 2 weeks of chemotherapy and transfusion of a total of 311 units (26 units of red blood cell [RBC], 26 units of fresh frozen plasma [FFP], 74 units of platelet concentrate $[\mathrm{PC}], 17$ units of platelet apheresis [PA], and 168 units of cryoprecipitate), the results of the coagulation test improved. On the 28th day after hospital admission, the patient was discharged. VAC, vincristine/doxorubicin/ cyclophosphamide; V, vincristine; FDP, fibrin/fibrinogen degeneration product. 
plasminogen activator inhibitor type 1. Virtually, all pathways that lead to the occurrence of DIC are driven by proinflammatory cytokines, such as interleukin-6, interleukin-1 $\beta$, interleukin-8, and tumor necrosis factor- $\alpha^{2)}$.

The pediatric patients with rhabdomyosarcoma complicated with DIC at diagnosis are rare. These cases were found in the review $^{5)}$ of 13 cases, published from 1978 to 2010 . Out of 11 patients with the involvement of BM, 10 cases in which the patients have recovered from the DIC received multiagent chemotherapy, and the recovery time from the DIC varied from 6 days to after 3 cycles of chemotherapy. Multivariate analysis revealed that the older age, male sex, primary tumor necrosis, and advanced tumor stage are the risk factors for $\mathrm{DIC}^{3)}$.

The prognosis of alveolar rhabdomyosarcoma stage IV with the involvement of BM is very poor, especially in the cases complicated by massive DIC ${ }^{5)}$. It is very important to administer chemotherapy early, with the aim of diminishing tumor-triggered procoagulant activity. There is a dilemma associated with this practice, because cytotoxic agents may aggravate DIC and may also suppress the BM. However, in an inoperable situation, administration of multidrug chemotherapy revealed to have an immediate beneficial effect, even if a measurable antitumor effect is not observed ${ }^{6)}$. In the case reported here, despite of the continuous bleeding at the postoperative site, administration of VAC chemotherapy at 4th HD was undertaken and the DIC showed signs of improvement after the 2nd week of chemotherapy.

In the cancer patients complicated with DIC, once the treatment directed at the primary disorder is initiated, the additional supportive therapy may be required in order to correct the coagulation abnormalities. Although the low levels of platelet and coagulation factors may increase the risk of bleeding, the plasma or platelet substitution therapy is not indicated on the basis of the laboratory findings alone, unless an active bleeding is present or the invasive procedures are required $^{7)}$. In our patient, the massive bleeding required as many as 311 units of blood component replacement (26 units of RBC, 26 units of FFP, 74 units of PC, 17 units of single donor platelets, and 168 units of cryoprecipitate).

Based on the concept that DIC is characterized by systemic activation of coagulation, anticoagulant therapy may be a principle approach in therapy. However, the safety and efficacy of this strategy in malignancy-associated DIC have never been studied in proper clinical settings ${ }^{2)}$. According to an experimental study, heparin therapy showed hopeful results in inhibiting the activation of coagulation in $\mathrm{DIC}^{8)}$. In the cases with obvious thromboembolic disease or where fibrin deposition predominates, heparin therapy could be considered. However, our patient did not receive heparin therapy and only received repeated transfusions, synthetic protease inhibitor, and substitution of antithrombin-III.

Clinically, DIC in malignancy has a less fulminant course than the types of DIC in sepsis and trauma. Particularly, solid tumors are the most common cause of chronic DIC. However, our case was unusual because of its acute presentation of severe DIC which was very difficult to control. The coagulopathy may be triggered by direct surgical manipulation of the tumor. By surgical trauma to the tumor blood vessels, exposure of the subendothelial TF causes the release of cytokines with their procoagulant activity. This can lead to the complication of acute DIC after the trauma to malignant cells ${ }^{9,10)}$.

In our case, early administration of chemotherapy and vigorous supportive care, including repeated transfusions, controlled the massive bleeding and DIC at the initial diagnosis. In conclusion, for the patients with rhabdomyosarcoma complicated with DIC, the early start of systemic chemotherapy to diminish the malignancy triggered procoagulant activity is important.

\section{Conflict of interest}

No potential conflict of interest relevant to this article was reported.

\section{References}

1. Bick RL, Arun B, Frenkel EP. Disseminated intravascular coagulation. clinical and pathophysiological mechanisms and manifestations. Haemostasis 1999;29:111-34.

2. Levi M. Disseminated intravascular coagulation in cancer patients. Best Pract Res Clin Haematol 2009;22:129-36.

3. Sallah S, Wan JY, Nguyen NP, Hanrahan LR, Sigounas G. Disseminated intravascular coagulation in solid tumors: clinical and pathologic study. Thromb Haemost 2001;86:828-33.

4. Barbui T, Falanga A. Disseminated intravascular coagulation in acute leukemia. Semin Thromb Hemost 2001;27:593-604.

5. Bien E, Maciejka-Kapuscinska L, Niedzwiecki M, Stefanowicz J, Szolkiewicz A, Krawczyk M, et al. Childhood rhabdomyosarcoma metastatic to bone marrow presenting with disseminated intravascular coagulation and acute tumour lysis syndrome: review of the literature apropos of two cases. Clin Exp Metastasis 2010;27:399407.

6. Weltermann A, Mitterbauer GJ, Mitterbauer M, Hattey E, Speiser W, Gisslinger H, et al. Disseminated intravascular coagulation (DIG) with massive hyperfibrinolysis in metastatic uterine cancer. Observations on the effects on the coagulopathy of various treatments (a case report). Wien Klin Wochenschr 1998;110:53-7.

7. Levi M, Opal SM. Coagulation abnormalities in critically ill patients. Crit Care 2006;10:222.

8. Pernerstorfer T, Hollenstein U, Hansen J, Knechtelsdorfer M, Stohlawetz P, Graninger W, et al. Heparin blunts endotoxin-induced coagulation activation. Circulation 1999;100:2485-90.

9. De Cicco M. The prothrombotic state in cancer: pathogenic mechanisms. Crit Rev Oncol Hematol 2004;50:187-96.

10. Teh RW, Tsoi DT. Acute disseminated intravascular coagulation in neuroendocrine carcinoma. Case Rep Oncol 2012;5:524-9. 